\title{
Transformation Conditions and Subloops In an Fe-Based Shape Memory Alloy Under Thermomechanical Loading
}

\author{
K. Tanaka, F. Nishimura and H. Tobushi* \\ Department of Aerospace Engineering, Tokyo Metropolitan Institute of Technology, Asahigaoka 6-6, \\ J-191 Hino/Tokyo, Japan \\ * Department of Mechanical Engineering, Aichi Institute of Technology, J-470-03 Toyota, Japan
}

\begin{abstract}
The martensite/austenite start and finish conditions, the transformation lines in the stress-temperature plane, are determined in an $\mathrm{Fe}-\mathrm{Cr}$-Ni-Mn-Si polycrystalline shape memory alloy under the mechanical and/or thermal loads. The transformation lines are show to be almost linear with nearly the same slope. The martensitic transformation zone and the reverse transformation zone are apart to each other, and the latter is very wide; $\mathrm{T}_{\mathrm{Af}}-\mathrm{T}_{\mathrm{As}} \approx 180 \mathrm{~K}$. Both the martensite start stress and the austenite start temperature are strongly dependent on the extent of prior transformations, the amount of preload and residual strain. The alloy performance is, therefore, fully contrary to the prediction of the thermodynamic theory and to the experimental result by Paskal and Monasevich in TiNi alloy. The effect of hold stress and temperature is measured to compare the transformation start condition with the yield condition in plasticity.
\end{abstract}

\section{INTRODUCTION}

The hysteretic behavior in the shape memory alloys during cyclic loading has intensively been investigated in different alloy systems [1-7]. The accumulation of the dislocations, therefore of the local stresses and strains were clearly observed during the process around the defects due to the repeated forward and backward sweep of the martensite/parent phase interfaces in the material [4,7]. The change in form of the full hysteresis loop and its shift and convergence to a limit loop are the topics of interest $[8,9]$.

The prior incomplete transformations has a strong effect on the form of the hysteresis loop, resulting in the stress-strain or strain-temperature subloops [10-15]. The study on the subloops is important when designing the shape memory devices because they always work under the small allowable mechanical and/or thermal loads so that there come no serious problems from the viewpoint of both the strength of alloys and the life of devices. Among others the martensite/austenite transformation start condition is the fundamental data to be investigated because it directly governs the size and shape of the subsequent hysteresis loops.

Paskal and Monasevich [10] have measured in a TiNi alloy that both the martensite and austenite start temperatures remain constant during the isostatic thermal loading, irrelevant to the extent of prior transformation. Tobushi et al. [11] also showed in a different TiNi alloy system that both the martensite and austenite start stresses are not influenced by the extent of prior transformation during the isothermal mechanical loading at the pseudoelastic temperature range. The case is, however, completely different from the alloy response of the $\mathrm{Cu}-\mathrm{Zn}-\mathrm{Al}$ and $\mathrm{Cu}-\mathrm{Al}-\mathrm{Mn}$ alloys by Amengual et al. [12], a $\mathrm{Cu}-\mathrm{Zn}-\mathrm{Al}$ alloy by Wei and Yang [13], an Fe-Mn-Si alloy by Robinson and McCormic [14] and an Fe-Mn-Si-Cr-Ni-Co alloy by Tan and Yang [15]. These data exhibit a clear dependence of the martensite/austenite start temperatures on the extent of prior transformations during the stress-free thermal loading.

According to the thermodynamic theory of materials in the process of martensitic transformation $[16,17]$, both the martensite start condition and the austenite start condition coincide with a linear line connecting the martensite start stress and the austenite start stress in the case of isothermal mechanical loading and the martensite start temperature and the austenite start temperature in the case of isostatic thermal loading. 
In this study the transformation condition is investigated in an $\mathrm{Fe}-9 \% \mathrm{Cr}-5 \% \mathrm{Ni}-14 \% \mathrm{Mn}-6 \% \mathrm{Si}$ polycrystalline shape memory alloy which is subjected to the thermomechanical loads. The martensite start stress in the stress-strain plane and the austenite start temperature in the strain-temperature plane are determined after the incomplete prior transformations. The cyclic effect on the subloops is not discussed here. The present results should, therefore, be understood to be the behavior in the alloys exhibiting a limit loop, after enough numbers of training cyclic loading.

\section{FUNDAMENTAL ALLOY PERFORMANCE}

The size of the specimen is $6 \mathrm{~mm}$ in diameter and $20 \mathrm{~mm}$ in gauge length. The transformation start and finish lines in the stress-temperature plane shown in Fig.1 [18-20] are important for the present study. The martensitic transformation still continues during the isothermal mechanical loading after the start of the macroscopic plastic deformation and is not completed even at the instance of fracture. The reverse transformation zone where the reverse transformation progresses is very wider, $A_{f}-A_{S} \approx 180 \mathrm{~K}$, compared to the case of TiNi alloys (See e.g. [6]). A thermomechanical loading of the type illustrated schematically in Fig. 2 is, therefore, the only case in this alloy to realize a successive martensitic and reverse transformations in the absence of macroscopic plastic deformation. The martensitic transformation progresses during the isothermal mechanical loading characterized by the hold temperature $T_{h}$ and the maximum stress $\sigma_{\max }$, while the reverse transformation undergoes during the subsequent isostatic thermal loading characterized by the hold stress $\sigma_{\mathrm{h}}$ and the maximum temperature $\mathrm{T}_{\max }$.

In order to eliminate the effect of cyclic loading, each specimen is subject before the test to 30 cycles of the following thermomechanical training: $\sigma_{\max }=350 \mathrm{MPa}, \mathrm{T}_{\mathrm{h}}=\mathrm{RT}(303 \mathrm{~K}), \mathrm{T}_{\max }=873 \mathrm{~K}>\mathrm{A}_{\mathrm{f}} \approx 457$ $\mathrm{K}$ and $\sigma_{\mathrm{h}}=0 \mathrm{MPa}$.

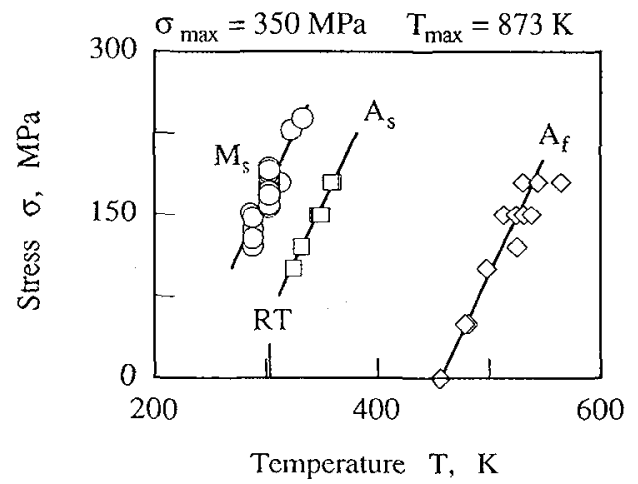

Fig.1 Transformation start and finish lines; $\sigma_{\max }=350 \mathrm{MPa}$.

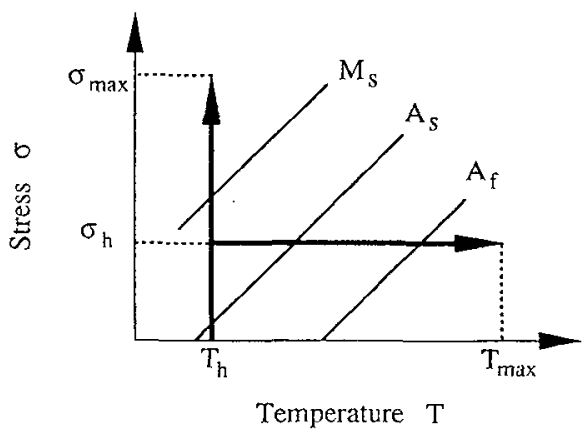

Fig.2 Thermomechanical loading path (Schematic).

\section{ISOTHERMAL MARTENSITE START STRESS}

The material performance during the following thermomechanical loading is first studied: $\sigma_{\max }=300$ $\mathrm{MPa}, \mathrm{T}_{\mathrm{h}}=\mathrm{RT}$ and $\sigma_{\mathrm{h}}=0 \mathrm{MPa}$ with the different values of $T_{\max }$. When $T_{\max }$ is lower than $A_{\mathrm{f}}$, the reverse transformation is incomplete during heating. The martensite start stress in the subsequent isothermal mechanical loading at $\mathrm{T}_{\mathrm{h}}=\mathrm{RT}$ would, therefore, be different from its initial value, $175 \mathrm{MPa}$, meaning that the martensite start stress might be dependent on $T_{\max }$, or rather on the whole prior transformation history in the strict sense. The result is summarized in Fig.3 together with the thermomechanical hysteresis during the successive loading/unloading-heating/cooling-reloading process. The martensite start stress determined as a $0.1 \%$ proof stress decreases with the extent of prior reverse transformation, which is an opposite result to what is predicted by the thermodynamic theory $[16,17]$ predicting that $\sigma_{\mathrm{Ms}}$ increases monotonically with the extent of prior reverse transformation. The present result is also different from the observation in a TiNi alloy by Tobushi et al. [11] who showed that $\sigma_{\mathrm{Ms}}$ was almost independent of the extent of prior reverse transformation. 
One could establish from the present data a relation

$$
\sigma_{\mathrm{Ms}}=\mu_{\sigma \xi}\left(\xi_{\mathrm{A} 0}\right)
$$

which directly connects $\sigma_{\mathrm{Ms}}$ with the amount of austenite $\xi_{\mathrm{A} 0}$ observed in the specimen just at the beginning of cooling. For the moment, however, $\sigma_{M s}$ as a function of $T_{\max }$ or of the residual strain $\varepsilon_{R}$ at the end of cooling process,

$$
\sigma_{\mathrm{Ms}}=\mu_{\mathrm{oT}}\left(\mathrm{T}_{\max }\right) \quad \text { or } \quad \sigma_{\mathrm{Ms}}=\mu_{\mathrm{oE}}\left(\varepsilon_{\mathrm{R}}\right),
$$

is enough to perform a further analysis of subloops during the incomplete transformations.

Figure 4 shows that the martensite start stress depends on the maximum stress $\sigma_{\max }$ in the isothermal loading process. The solid circles denote the yield stresses determined at RT with a single specimen in the reloading processes after mechanical loading/unloading, while the thin curve represents the original stress-"plastic" strain curve during simple monotone loading at RT. The difference of these two curves stems simply from the definition of the yield stress as in conventional plasticity. The iso- $\sigma_{\max }$ curves finally approach to the yield stress curve, the dotted curve, with the larger $\varepsilon_{R}$. The point on the yield stress curve means, therefore, the elastic unloading/loading without transformation.

The conclusion seems to be as follows:

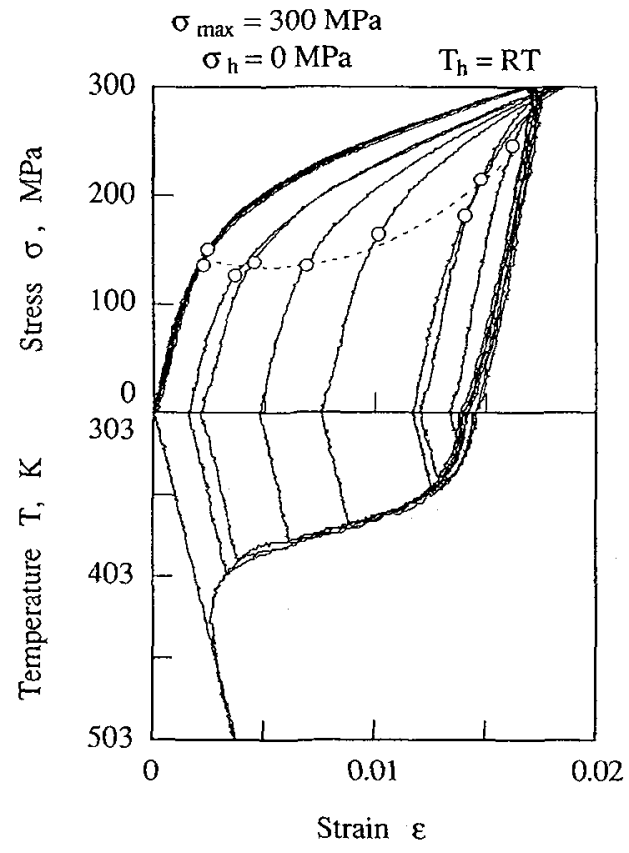

Fig.3 Martensite start stress after incomplete reverse transformation. Martensite start stress can be uniquely determined from the values of residual strain after cooling $\varepsilon_{R}$ as well as of the maximum stress during loading $\sigma_{\max }$;

$$
\sigma_{\mathrm{Ms}}=\mu_{\sigma \varepsilon}\left(\varepsilon_{\mathrm{R}}, \sigma_{\max }\right)
$$

which is a generalization of Eqs.(1) and (2) since a one-to-one relation could be expected between $\varepsilon_{R}$ and $\xi_{\mathrm{A} 0}$.

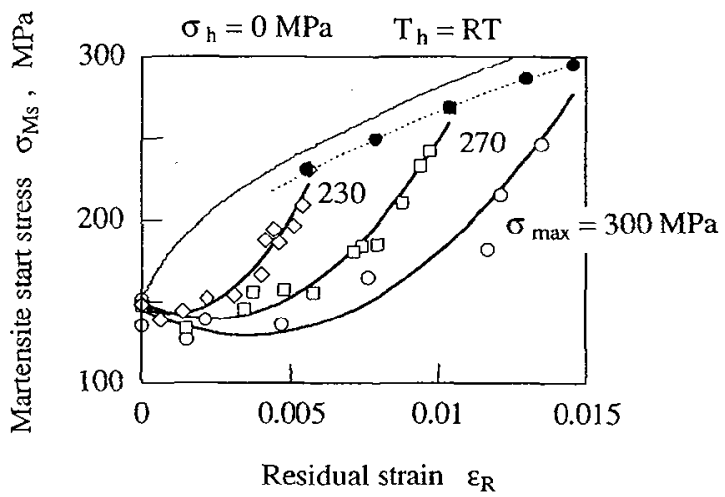

Fig.4 Dependence of martensite start stress on prior transformations.

\section{ISOSTATIC AUSTENITE START TEMPERATURE}

When the alloy specimen is subjected to the thermomechanical loads; $T_{h}=R T, \sigma_{h}=0 \mathrm{MPa}$ and $T_{\max }$ $=873 \mathrm{~K}>A_{f}$ with the different values of $\sigma_{\max }$, an austenite start temperature $T_{A s}$ is measured from each strain-temperature curve in the isostatic heating process. The results summarized in Fig.5 clearly show that $\mathrm{T}_{\mathrm{As}}$ determined in the isothermal mechanical loading process decreases nonlinearly with the extent of prior martensitic transformation. The case is different from the result in the TiNi alloys with constant $\mathrm{T}_{\text {As }}$ observed by Paskal and Monasevich [10] and Tobushi et al. [11]. The thermodynamic prediction [16,17] does not explain the qualitative tendency of the present data. The experimental results reported by the metallurgists [12-15] in various alloys seem to support the data although they all carried out their experiments, not in the thermomechanical process as in the present case but, in the simpler isostatic heating/cooling process. 


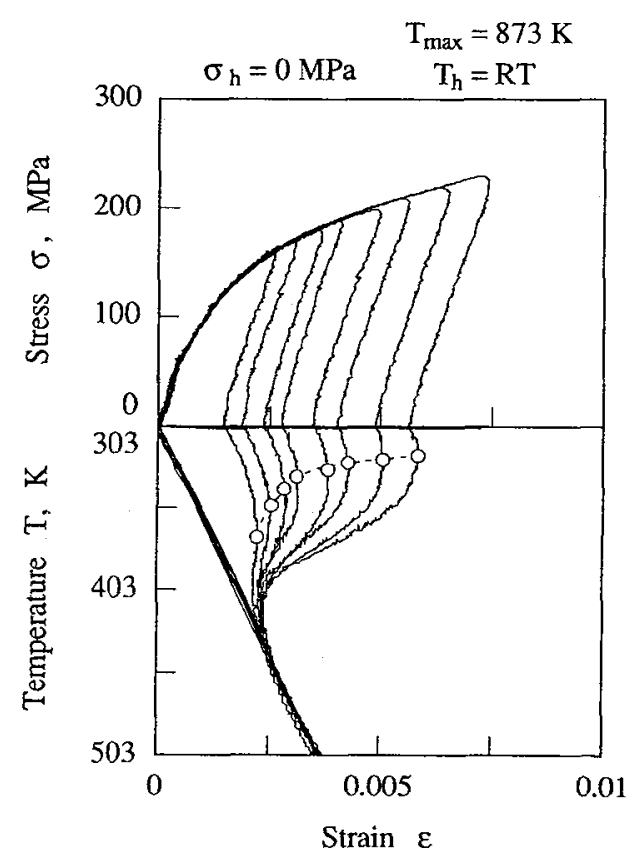

Fig.5 Austenite start temperature after incomplete martensitic transformation.

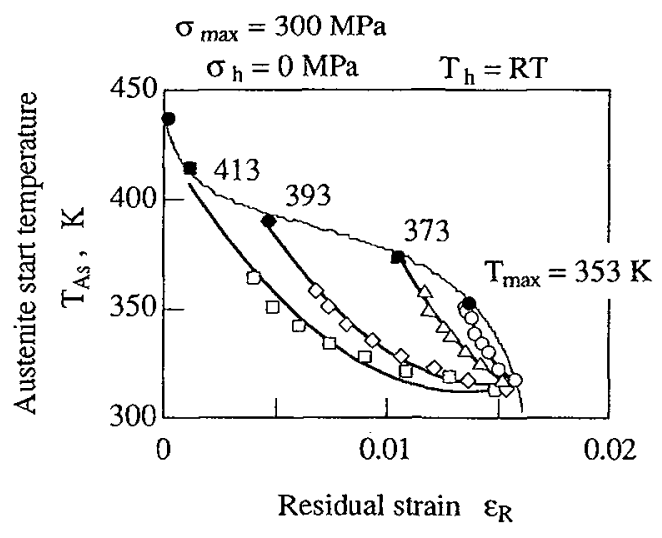

Fig.6 Dependence of austenite start temperature on prior transformations.

The same type of test leading to Fig.4 is carried out to reveal the effect of the prior transformations on the austenite start temperature (cf. Fig.6). The thin curve in the figure represents the strain-temperature out-put during a thermomechanical loading; $\sigma_{\max }=300 \mathrm{MPa}, \mathrm{T}_{\mathbf{h}}=$ $\mathrm{RT}, \mathrm{T}_{\max }=873 \mathrm{~K}$ and $\sigma_{\mathrm{h}}=0 \mathrm{MPa}$, while the solid circles show the austenite start temperature determined with a single specimen during a successive heating/cooling under $\sigma_{\mathrm{h}}=0 \mathrm{MPa}$ after

an isothermal mechanical loading/unloading; $\sigma_{\max }=300 \mathrm{MPa}, \mathrm{T}_{\mathrm{h}}=\mathrm{RT}$. The conclusion is that the austenite start temperature can be well specified both by the extent of prior martensitic and reverse transformations as

$$
\mathrm{T}_{\mathrm{As}}=\alpha_{\mathrm{TE}}\left(\varepsilon_{\mathrm{R}}, \mathrm{T}_{\max }\right) \text {. }
$$

A short comment on the austenite finish temperature $T_{A f}$ [20]: When the austenite finish temperature is measured, during the tests leading to Fig.6, for each strain-temperature curve in the isostatic heating, it, contrary to the case of the austenite start temperature, depends solely on $\sigma_{\max }$ and increases with the extent of prior martensitic transformation.

\section{EFFECT OF HOLD STRESS AND TEMPERATURE}

The effect of the hold stress $\sigma_{\mathrm{h}}$ on the result in Fig.6 is illustrated in Fig.7 with the open circles for the condition $\sigma_{\mathrm{h}}=70 \mathrm{MPa}$ and $\mathrm{T}_{\max }=413 \mathrm{~K}$ and with the open squares for the condition $\sigma_{\mathrm{h}}=0 \mathrm{MPa}$ and $T_{\max }=381 \mathrm{~K}$. It is worth noting that the maximum temperature $T_{\max }=413 \mathrm{~K}$ under $\sigma_{\mathrm{h}}=70 \mathrm{MPa}$ and $\mathrm{T}_{\max }=381 \mathrm{~K}$ under $\sigma_{\mathrm{h}}=0 \mathrm{MPa}$ are both about $87 \mathrm{~K}$ higher than the austenite start line. Provided that the iso-fraction lines are parallel to the austenite start and finish lines in the reverse transformation zone, meaning that the extent of reverse transformation could be determined, irrelevant to the value of the hold stress $\sigma_{\mathrm{h}}$, by the excess temperature above the austenite start temperature, the two series of experimental data in Fig. 7 would coincide. Figure shows the inspection is not the case.

From the isothermal stress-strain curves determined at the several hold temperatures $T_{h}$, the iso-transformation strain $\varepsilon_{R 0}$ lines can be constructed as given in Fig.8. The iso- $\varepsilon_{R 0}$ lines, which correspond to the initial and subsequent yield surfaces in plasticity, are linear but not parallel. The specimens loaded isothermally up to the $\varepsilon_{R_{0}}=0.01$ line at several hold temperatures $T_{h}$ exhibits the 
different values of the martensite start stress in the subsequent heating-cooling-reloading process. The result is illustrated in Fig.9 showing a clear effect of the hold temperature $T_{h}$. The figure is replotted in Fig. 10 by employing $\varepsilon_{R}$ as a parameter. The iso- $\varepsilon_{R}$ lines, corresponding to the subsequent yield surfaces during reloading, are again linear lines in the stress-tempcrature plane, but not parallel. The way of shifting of the "subsequent transformation line" is not simple especially at the start of the transformation. When the transformation progresses the transformation line coincides with the initial line as indicated at each temperature by the end point of the arrow.

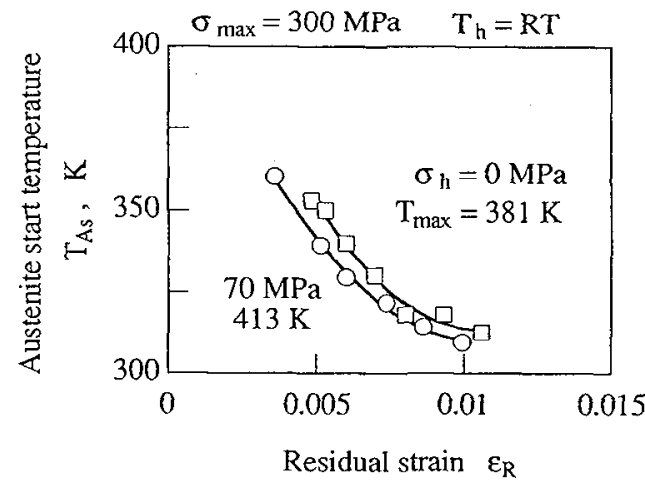

Fig.7 Effect of hold stress on austenite start temperature.

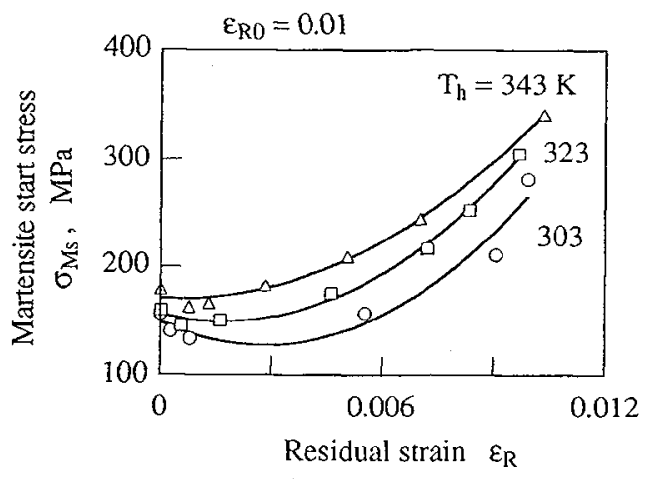

Fig.9 Effect of hold temperature on martensite start stress.

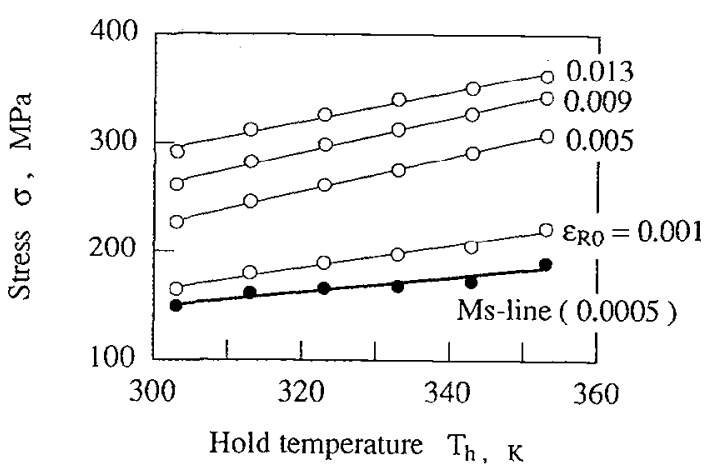

Fig.8 Initial/subsequent transformation lines.

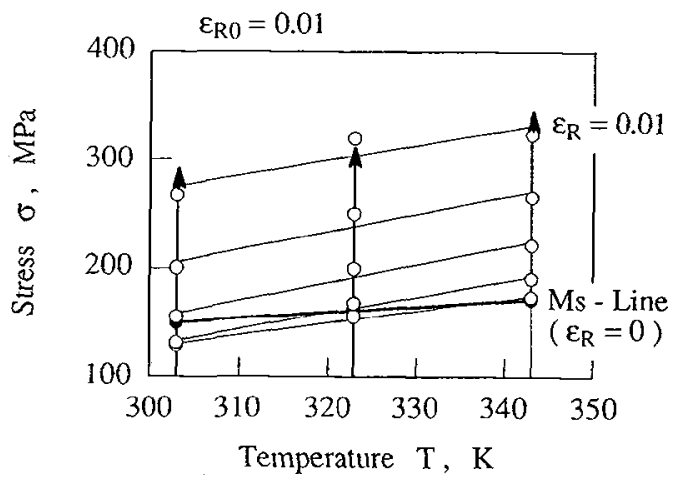

Fig.10 Subsequent transformation lines during reloading.

\section{A REMARK ON TRANSFORMATION START LINES}

Thermodynamics of martensitic transformation [21-23] tells us that the transformation starts at a material point when a thermomechanical condition

$$
\Delta \mathrm{G}(\boldsymbol{\sigma}, \mathrm{T})=-\mathrm{G}_{0},
$$

holds at the point for the driving force $\Delta G(\sigma, T)$, which is composed of the chemical part and the mechanical part, where $\sigma$ and $T$ stand for the stress tensor and the temperature measured at just the point, and $G_{0}>0$ the threshold value. The decomposition of the local stress tensor $\sigma$ into the applied stress and the internal stress term which is induced due both to the birth of the martensite phase in the parent phase and to the interaction of the martensite plates is a theme to be intensively investigated in micromechanics [24]. The condition can be understood to represent a transformation start plane in the $\sigma$ - $T$ space, which can be reduced to the transformation start lines discussed in the present study. 


\section{ACKNOWLEDGMENTS}

Part of this work was financially supported by the Special Research Fund/ Tokyo Metropolitan Government as well as by the Grant-in-Aid for Scientific Research (No.05650087) through the Ministry of Education, Science and Culture, JAPAN. The authors extend their gratitude to the Steel Research Center/ NKK Corporation for supplying the alloy specimens.

\section{REFERENCES}

[1] Li,J.C. and Ansel,G.S., Metall. Trans. A 14A (1983) 1293-1297.

[2] Sade,M., Halter,K. and Hornbogen,E., Z. Metallkde. 79 (1988) 487-491.

[3] Contardo,L. and Guénin, G., Acta Metall. Mater. 38 (1990) 1267-1272.

[4] Miyazaki,S. and Otsuka,K., ISIJ Int. 29 (1989) 353-377.

[5] Xu,Hubin and Tan Shuson, Scripta Metal. Mater. 25 (1991) 1507-1511.

[6] Tobushi,H., Iwanaga,H., Tanaka,K., Hori,T. and Sawada,T., Continuum Mech. Thermodyn. 3 (1991) 79-93.

[7] Miyazaki,S., Imai,T., Igo,Y. and Otsuka,K., Metall. Trans. A 17A (1986) 115-120.

[8] Duerig,T.W., Melton,K.N., Stöckel,D. and Wayman,C.M. (eds.), Engineering Aspects of Shape Memory Alloys (Butterworth-Heinemann, London, 1990).

[9] Funakubo,H. (ed.), Shape Memory Alloys (Gordon and Breach Science Publishers, New York, 1987).

[10] Paskal,Yu.I. and Monasevich,L.A., Phys. Met. Metall. 53 (1981) 95-99.

[11] Tobushi,H., Iwanaga,H., Tanaka,K., Hori,T. and Sawada,T., JSME Int. J., Ser.I 35 (1992) 271-277.

[12] Amengual,A., Cesari,E. and Seguí,C., Martensitic Transformations '92 (Monterey Institute of Advanced Studies, Carmel, 1993) pp.377-382.

[13] Wei,Zhongguo and Yang,Dazhi, Scripta Metall. 22 (1988) 1245-1249.

[14] Robinson,J.S. and McCormick,P.G., Material Sci. Forum 56-58 (1990) 649-654.

115] Tan,Shiming and Yang,Shiwei, Scripta Metall. Mater. 27 (1992) 229-232.

[16] Müller,I., Continuum Mech. Thermodyn. 1 (1989) 125-142.

[17] Raniecki,B., Lexcellent,C. and Tanaka,K., Arch. Mech. 44 (1992) 261-284.

[18] Tanaka,K., Hayashi,T., Aida,Y. and Tobushi,H., J. Intell. Material Syst. Struct. 4 (1993) 567-573.

[19] Tanaka,K., Hayashi,T., Nishimura,F. and Tobushi,H., J. Materials Engng. Performances 3 (1994) 135-1431.

[20] Tanaka,K., Hayashi,T., Fischer,F.D. and Buchmayr,B., Z. Metallkd. 85 (1994) 122-126.

[21] Ortín,J. and Planes,A., Acta Metall. 36 (1988) 1873-1839.

[22] Kaufman,L. and Hillett,M., Martensite (ASM International, 1992) Chap.4.

[23] Fischer,F.D., Berveiller,M., Tanaka,K. and Oberaigner,E.R., Arch. Appl. Mech. 63 (1994) 54-85.

[24] Marketz,F. and Fischer,F.D., MECAMAT 93, Int. Seminar on Micromechanics of Materials (Editions Eyrolles, Paris, 1993) pp.258-269. 